\title{
Influence of Polymer Coating on Device Properties of Carbon Nanotube Field-Effect Transistors
}

\author{
Shinya Aikawa ${ }^{1}$, Taiki Inoue ${ }^{1}$, Erik Einarsson ${ }^{1,2}$, Shohei Chiashi $^{1}$, and Shigeo Maruyama ${ }^{1}$ \\ ${ }^{1}$ Department of Mechanical Engineering, The Univ. of Tokyo \\ 7-3-1 Hongo, Bunkyo-ku, Tokyo 113-8656, Japan \\ Phone: +81-3-5841-6421 E-mail: maruyama@photon.t.u-tokyo.ac.jp \\ ${ }^{2}$ Global COE for Mechanical Systems Innovation, The Univ. of Tokyo \\ 7-3-1 Hongo, Bunkyo-ku, Tokyo 113-8656, Japan
}

\section{Introduction}

Flexible and transparent field-effect transistors (FETs) based on organic or inorganic materials are now one of the most attractive research objects because they have potential to bring innovation in conventional electronic device systems. Recently, high-performance flexible integrated circuits using carbon nanotube thin-film transistors (CNT-TFT) have been developed [1-3]. These devices have shown excellent device properties, however, both optical transparency and mechanical flexibility are limited due to the use of opaque metal electrodes and brittle oxide dielectrics. To realize extremely flexible and highly transparent devices, we suggest that robust and pliable materials such as carbon electrodes (carbon nanotube networks or graphene), polymer dielectrics and thin plastic substrates should be used for device components.

In our previous work, we used as-grown single-walled carbon nanotubes (SWNTs) as both electrode and channel material, and poly(vinyl alcohol) (PVA) employed as dielectric layer and substrate, thus realizing extremely flexible (radius of curvature, $R=1.0 \mathrm{~mm}$ ) and transparent (more than $80 \%$ transmittance in the visible range) CNT-FETs [4]. On the other hand, the conduction type of the CNT-FETs changed from p-type to ambipolar behavior by poly(vinyl alcohol) (PVA) coating. Singh et al. also reported such ambipolar conversion using PVA as dielectric layer in organic FETs [5]. However, the conversion mechanism has not yet been clarified.

Here, we try to understand why carrier transport behavior of CNT-FET can be changed by polymer coating and also to control the device properties (e.g. p/n type, threshold voltage, etc.). To control the polarity of FETs, as well as to realize high-performance flexible electronic devices, it is very important to clarify interfacial interaction between CNTs and polymers.

\section{Experiments}

The fabrication process of CNT-FETs is as follows. Patterned catalyst deposition (Co thickness: $0.2 \mathrm{~nm}$ ) was performed on a Si substrate $\left(t_{\mathrm{ox}}: 600 \mathrm{~nm}\right)$ by conventional photolithography and lift-off processes. SWNTs were synthesized by alcohol catalytic chemical vapor deposition (ACCVD) [6]. PVA solution with $10 \%$ concentration was dropped onto the channel region as shown in Fig. 1 and then dried on a hot plate. The devices were characterized using a semiconductor parameter analyzer (Agilent 4156C) at room temperature under ambient conditions.

\section{Results and discussion}

Figure 2 shows typical transfer characteristics of an as-fabricated CNT-FET. The $I_{\mathrm{D}}-V_{\mathrm{GS}}$ curve indicates that the device is unipolar p-type conduction with an on/off ratio of $10^{4}$. The calculated field-effect mobility $\left(\mu_{\text {eff }}\right)$ is 20.9 $\mathrm{cm}^{2} \cdot \mathrm{V}^{-1} \cdot \mathrm{s}^{-1}$ in the forward sweep direction using the following equation (parallel plate model):

$$
\mu_{\mathrm{eff}}=\frac{I_{\mathrm{D}}}{\partial V_{\mathrm{GS}}} \frac{L}{W} \frac{1}{C_{i}\left(V_{\mathrm{GS}}-V_{\mathrm{th}}\right)}
$$

where, $\partial I_{\mathrm{D}} / \partial V_{\mathrm{GS}}=g_{\mathrm{m}}$ is the transconductance, $L$ the channel length, $W$ the channel width, $C_{i}$ the capacitance per unit,

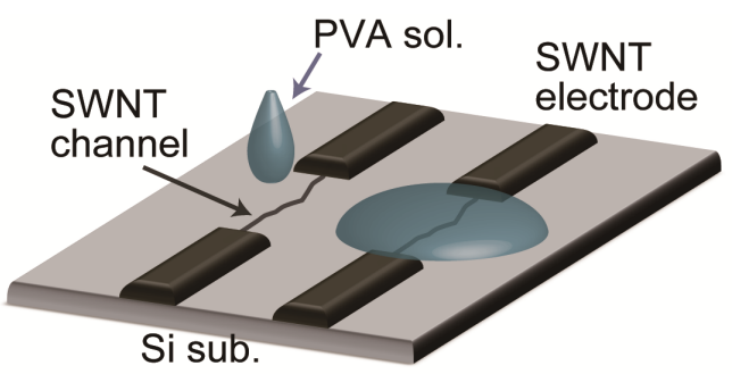

Fig. 1 Schematic of CNT-FET device structure.

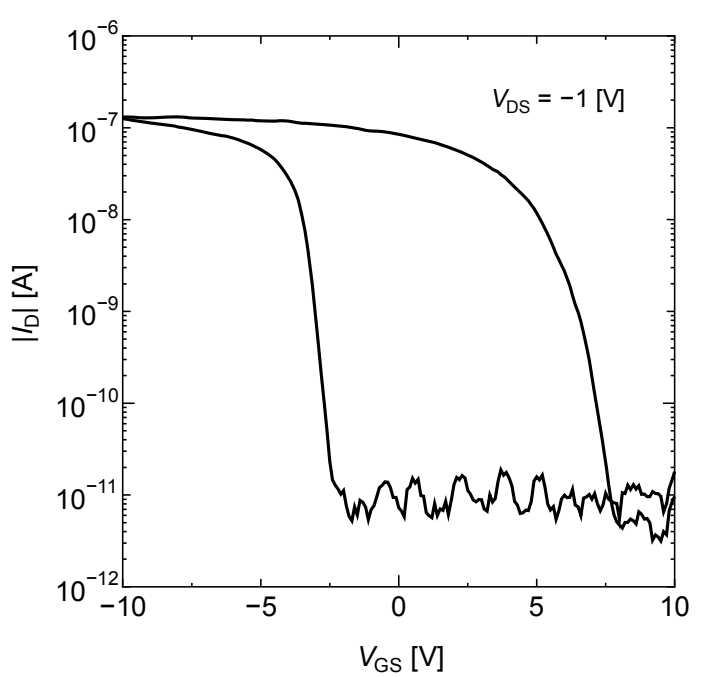

Fig. 2 Typical transfer characteristics of as-fabricated CNT-FET. 
and $V_{\text {th }}$ the threshold voltage. For this calculation, a device with $L=10 \mu \mathrm{m}$ and $W=5 \mu \mathrm{m}$, and the estimated values of $g_{\mathrm{m}}=42.2 \mathrm{nS}, C_{i}=5.76 \mathrm{nF} / \mathrm{cm}^{2}$ (dielectric constant of $\mathrm{SiO}_{2}$ : 3.9 ) and $V_{\text {th }}=-2.9 \mathrm{~V}$ were used. The mobility is as good as solution-processed CNT-TFTs, which use separated semiconducting SWNTs for the channels [7,8], even though the number of SWNT channels in our devices is only one or two. This might be due to good electrical contact between SWNT electrodes and the SWNT channels [9].

Figure 3 demonstrates the transfer characteristics before and after PVA-coating on the same CNT-FET. The uncoated device shows typical p-type conduction, whereas the PVA-coated CNT-FET shows ambipolar behavior and $V_{\text {th }}$ was slightly shifted in the negative direction. We have measured more than 5 devices and all of them showed a similar tendency. The reason for this change might be attributed to the positive fixed surface charges of PVA layer as shown in Fig. 4(a). Due to these charges, band bending in the semiconductor channel occurrs and electrons can be injected into the SWNT channel at positive gate voltages (Fig. 4(b)). The estimated charge density is about $10^{11} \mathrm{~cm}^{-2}$. This is much lower for achieving unipolar n-type conduc-

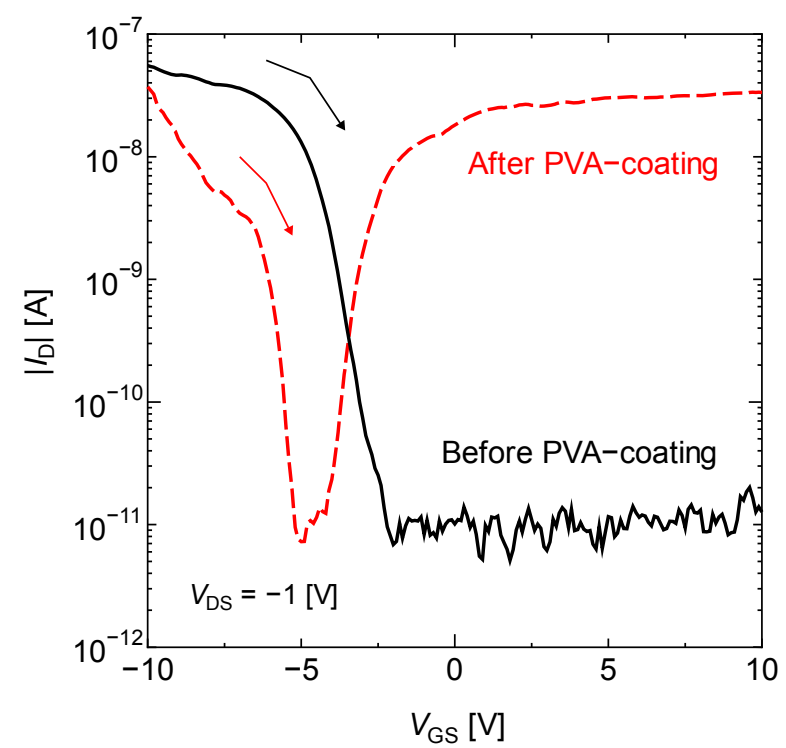

Fig. 3 Transfer characteristics before and after PVA-coating on the same CNT-FET. (a)

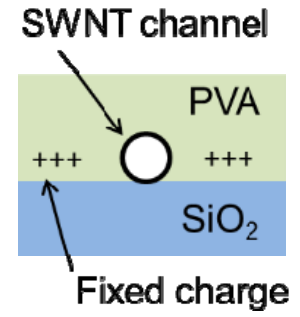

(b)

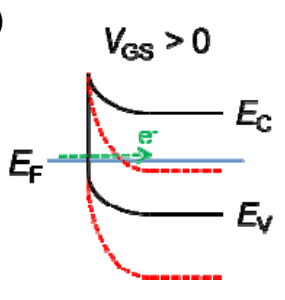

Fig. 4 (a) Schematic cross-section diagram of $\mathrm{PVA} / \mathrm{SiO}_{2}$ interface. (b) Illustration of band bending due to the fixed surface charges of PVA (dashed red lines). The solid black lines indicate normal case (without fixed charges).
Table I Device parameters before and after PVA- coating.

\begin{tabular}{llll}
\hline PVA coating & $\mu_{\mathrm{eff}}\left[\mathrm{cm}^{2} \cdot \mathrm{V}^{-1} \cdot \mathrm{s}^{-1}\right]$ & $g_{\mathrm{m}}[\mathrm{nS}]$ & $V_{\text {th }}[\mathrm{V}]$ \\
\hline Before & 3.28 & 15.1 & -3.4 \\
After & 1.33 & 3.8 & -5.3 \\
\hline
\end{tabular}

tion [10], but is enough for ambipolar conversion. Typical device parameters before and after PVA-coating are summarized in Table I.

\section{Summary}

We fabricated CNT-FETs, in which SWNTs were employed as both electrode and channel, on Si substrates. The device with good performance $\left(\mu_{\text {eff }}=20.9 \mathrm{~cm}^{2} \cdot \mathrm{V}^{-1} \cdot \mathrm{s}^{-1}\right)$ was obtained. Furthermore, the transfer characteristics clearly showed that carrier transport behavior is converted from unipolar p-type to ambipolar conduction after PVA-coating. We hypothesize that this may be due to fixed surface charges of the PVA layer. The detailed discussion on the conversion mechanism will be presented.

\section{Acknowledgements}

Part of this work was financially supported by Grant-in-Aid for Scientific Research (19054003 and 22226006), and Global COE Program 'Global Center for Excellence for Mechanical Systems Innovation'. This work was also supported by the VLSI Design and Education Center (VDEC), The University of Tokyo, in collaboration with Cadence Corporation.

\section{References}

[1] Q. Cao, H. S. Kim, N. Pimparkar, J. P. Kulkarni, C. Wang, M. Shim, K. Roy, M. A. Alam and J. A. Rogers, Nature 454, 495 (2008).

[2] D. M. Sun, M. Y. Timmermans, Y. Tian, A. G. Nasibulin, E. I. Kauppinen, S. Kishimoto, T. Mizutani and Y. Ohno, Nat. Nanotechnol. 6, 156 (2011).

[3] C. Wang, J. C. Chien, K. Takei, T. Takahashi, J. Nah, A. M. Niknejad and A. Javey, Nano Lett. 12, 1527 (2012).

[4] S. Aikawa, E. Einarsson, T. Thurakitseree, S. Chiashi, E. Nishikawa and S. Maruyama, Appl. Phys. Lett. 100, 063502 (2012).

[5] T. B. Singh, F. Meghdadi, S. Günes, N. Marjanovic, G. Horowitz, P. Lang, S. Bauer and N. S. Sariciftci, Adv. Mater. 17, 2315 (2005).

[6] S. Maruyama, R. Kojima, Y. Miyauchi, S. Chiashi and M. Kohno, Chem. Phys. Lett. 360, 229 (2002).

[7] M. S. Arnold, A. A. Green, J. F. Hulvat, S. I. Stupp and M. C. Hersam, Nat. Nanotechnol. 1, 60 (2006).

[8] N. Rouhi, D. Jain, K. Zand and P. J. Burke, Adv. Mater. 23, 94 (2011).

[9] S. Aikawa, R. Xiang, E. Einarsson, S. Chiashi, J. Shiomi, E. Nishikawa and S. Maruyama, Nano Res. 4, 580 (2011).

[10] N. Moriyama, Y. Ohno, K. Suzuki, S. Kishimoto and T. Mizutani, Appl. Phys. Express 3, 105102 (2010). 